\title{
Rate Adaptation with NAK-Aided Loss Differentiation in 802.11 Wireless Networks
}

\author{
Anne N. Ngugi*, Yuanzhu Peter Chen* and Qing $\mathrm{Li}^{\dagger}$ \\ ${ }^{*}$ Computer Science Department \\ Memorial University of Newfoundland \\ St. John's, Canada \\ ${ }^{\dagger}$ School of Economic Information Engineering \\ Southwestern University of Finance and Economics, Chengdu, China
}

\begin{abstract}
The Physical Layer of the IEEE 802.11 standard family provides a set of different modulation and coding schemes and, thus, a multitude of data rates. However, the Standard itself does not specify a mechanism to select adaptively among these data rates to improve the network throughput. An efficient rate adaptation scheme should aim to improve channel utilization through selection of optimal data rates that suits current channel conditions. This paper proposes a rate adaptation technique that: 1) exploits the RSS of received DATA frame to recommend higher data rate for subsequent transmissions, 2) distinguishes causes of a frame loss by recording RSS of a CTS/ACK frame to predict if the channel quality is deteriorating or not, and 3) utilizes a NAK-frame to diagnose frame losses due to channel fading. Our scheme, dubbed as Differential Rate Adaptation with NAKAssisted Loss Differentiation (DRANLD), is simulated using ns-2 and shown to adapt well to rapidly fluctuating channels.
\end{abstract}

\section{INTRODUCTION}

The IEEE 802.11 PHY Specifications provide any compliant device a multi-rate capability, but the IEEE 802.11 MAC Specification does not specify any mechanism to utilize this capability [1]. It is up to the wireless device vendors to come up with interfaces to use this multi-rate capability. The ability to select one out of multiple available transmission rates dynamically is referred to as rate adaptation or link adaptation. Any rate adaptation scheme ought to have a mechanism to estimate the current channel conditions and a plan that adapts the data rate according to the channel estimation. There has been a fair amount of research on this issue lately.

Existing rate adaptation schemes can broadly be categorized into two classes, i.e. open loop and closed loop approaches. In open loop approaches, the sender makes decision based on its own perception of channel conditions, such as success or failure of previous DATA frame transmission or the signal strength of a received ACK frame. Closed loop approaches depend on the receiver to estimate the channel quality and to communicate this information back to the sender to select the appropriate data rate. These two categories can further be classified according to the technique used by the sender or receiver to estimate the channel quality. Some of the techniques used to estimate channel quality include:

- Transmission history - A node can obtain link level information by maintaining statistics, such as the Frame Error Rate (FER), number of acknowledged frames, and the achieved throughput for the transmitted frames. This approach is necessarily passive and can be slow to adapt to the actual channel changes, and may even introduce oscillation in moderately changing channel conditions. Some schemes that utilize this technique include [2], [3].

- PHY information - A device can also estimate data rate by analyzing SNR or the Received Signal Strength (RSS) of a received DATA frame [4]-[8].

- Use of RTS/CTS - Beyond the original use of RTS/CTS to address the hidden terminal problem in wireless channels, the RTS/CTS mechanism can also be used to assess channel quality and to estimate the effective data rate [3][5], [8], [9].

- Frame burst - A node should take advantage of good channel quality when it lasts [5], [6], [8]. That is, when a sender captures the medium, it holds on to it for the duration of time equivalent to the time required to transmit a single frame at the basic data rate. If the channel quality permits higher data rate than the basic rate, then the sender can send as many frames as it can at this higher rate during the burst.

- Beacon frame - A beacon frame is periodically transmitted by an access point to allow mobile stations to find and join a network. Rate adaptation can utilize the mandatory periodic IEEE 802.11 beacon instead of RTS/CTS for rate estimation [7].

- Loss differentiation - Most rate adaptation protocols implicitly assume that frame loss is caused by bad channel conditions and fallback to a lower data rate upon DATA loss [2]. However, in dense multi-hop wireless networks, collision can also cause frame loss, and reducing data rate in this case could further degrade the network performance by extending both transmission and collision time of the DATA frame. Thus, smarter rate adaptation schemes diagnose the actual cause of frame loss before deciding to decrease retransmission rate. Such schemes [3], [6], [7], [9] are said to be loss differentiating, or LD for short.

- Fragment size adjustment - The rationale behind reducing frame fragmentation length is that, in a collision prone environment, the probability of successful delivery of a shorter frame is much higher than that of long 
frame. However, in a degraded channel, the probability of successful delivery of a shorter frame is not significantly higher than that of longer frame [6], [10]. This inference is useful in distinguishing different causes of frame losses.

- Contention window control - An efficient lossdifferentiating mechanism should double the backoff window if frame loss is due to collision but freeze the contention window if frame loss is due to link errors [6], [7].

- Negative acknowledgement - A MAC DATA frame consists of two functional parts: the header and the payload. The header contains the frame type, source and destination address, among other things. If multiple stations transmit at the same time, then both the MAC header and the body of the DATA frame will be destroyed. If there is frame loss due to link errors, there is a chance that the receiver will receive the MAC header but the body will be corrupted. From the header, the receiver can gather the source address and send back a NAK frame which indicates frame loss due to link errors [9]. This can be achieved using an additional frame header checksum field.

This work incorporates two features into our earlier work, DRA [8].

- An RSS-based LD mechanism. This LD mechanism is motivated by the observation in [11], [12], that the Receive Signal Strength (RSS) at the sender and receiver are strongly correlated, which means the sender can use its own RSS measurements to make channel quality judgement at the receiver. This correlation has been observed using testing environment consisting of MICA2 motes.

- A NAK-aided loss-differentiation mechanism, which provides means for the receiver to communicate its channel quality perception back to the sender. It also alleviates the possibility of using outdated CTS/ACK's RSS value to infer link errors or collision.

The remaining sections of this paper are organized as follows. Section II describes rate adaptation schemes whose design techniques are most related to our work. In Section III, we describe the design features of our proposed NAK-aided loss differentiating scheme, DRANLD. Section IV evaluates the performance of DRANLD and last, in Section V, we conclude our work and provide future research directions in rate adaptation.

\section{RELATED WORK}

\section{A. OAR}

Opportunistic Auto Rate (OAR) [5] is an SNR-based rate adaptation scheme built on top of RBAR [4] to exploit good channel quality by transmitting multiple back-to-back DATA frames. When an OAR sender captures the channel, it holds it for the period equivalent to time $t$ required to transmit a single frame at the lowest data rate (basic rate). If the channel conditions allow for a higher rate than the basic rate, then sender can opportunistically send as many frames as it can, at this higher rate within time $t$. OAR allows nodes with high channel quality to take advantage of it by transmitting more DATA frames than those with low channel quality while ensuring that all nodes are granted the same channel access time $t$. OAR improves RBAR significantly by allowing a burst of DATA frames to be preceded by a single RTS/CTS handshake. However, it assumes a constant channel condition during the burst transmission time and may not respond to rapid channel changes.

\section{B. $L D-A R F$}

LD-ARF [9] uses two LD methods depending on whether RTS/CTS is enabled or not. If RTS/CTS is enabled and CTS is successfully received but the ACK frame is missing, the DATA loss is said to be caused by fading channel quality, and a lower rate is used for retransmission. However, if CTS is missing, the DATA loss is caused by collision and the current data rate is to be used for retransmission. On the other hand, if RTS/CTS is disabled, a garbled DATA frame triggers the receiver to transmit a NAK if the MAC header of the DATA frame can be reconstructed correctly. The argument behind this is that, even if the channel conditions are so bad that the DATA frame could not be decoded, still the MAC header can be recovered thanks to its smaller size.

LD-ARF is mainly a statistic-based rate adaptation scheme, which makes most of its design features different from our proposed scheme, DRANLD. Therefore, although it uses NAK for loss differentiation, we shall not compare its performance with DRANLD.

\section{DRANLD DESIGN}

In this section, we discuss the three main features that make up our rate adaptation scheme dubbed as, Differential Rate Adaptation with NAK-Aided Loss Differentiation (DRANLD). In general, DRANLD is based on the design of OAR, where a single RTS/CTS exchange leads a burst of multiple DATA/ACK (or NAK) separated by SIFS. The module to determine if the sender should increase data rate in event of receiving ACK is the core of our previous work, DRA [8], which is reviewed in Section III-A. In event of frame loss, that is, either missing ACK or receiving NAK, the sender needs to retransmit the fragment immediately. Depending on if the sender determines whether the loss was caused by link error or collision, it may choose to retransmit the fragment at the same rate or reduce it. The two mechanisms to support such a decision are described in Sections III-B and III-C. The entire control logic of a DRANLD sender is depicted in Section III-D. In this section, we assume that there are a set of data rates that a sender can choose from, $\left\{r_{1}, r_{2}, \ldots, r_{k}\right\}$, in an increasing order.

\section{A. Differential Rate Adaptation - DRA}

DRA uses a single RTS/CTS frames exchange to precede a burst of DATA/ACK pairs transmitted within burst time $t$, 
which is usually equivalent to time required to successfully transmit a single DATA frame at the basic rate. A DRA receiver, like OAR, communicates the initial data rate back to the sender via a CTS frame. In addition, it utilizes the unused retry bit of an ACK frame to recommend a higher data rate in order to increase the number of subsequent DATA fragments in the burst. Upon reception of a DATA frame, the receiver compares the RSS of the moment to the current data rate. If this RSS supports a higher data rate, the returning ACK's retry bit is set to 1 ; else, it is set to 0 . When the sender receives an ACK, it checks the ACK's retry flag value. If it is set to 1 , the sender adjusts its subsequent transmission rate to the next available higher rate. Otherwise, the next DATA frame is transmitted at the current data rate. If the ACK frame is missing, then the sender retransmits the same DATA frame at the current rate, and does so until either it is successfully acknowledged, the allowed retransmit limit is exhausted, or its burst time is up.

\section{B. RSS-Based Loss Differentiation}

When a fragment is lost, it should be retransmitted at the same or a lower data rate, depending on its causes. Thus, we incorporate a simple but powerful RSS-based LD mechanism, which is motivated by the observation made in [11], [12], i.e. the RSS at the sender and receiver are strongly correlated. This means that a sender can use its own RSS measurements to make channel quality judgement at the receiver.

A sender stores two variables to help to distinguish the cause of a DATA fragment loss, i.e. an RSS measurement $R_{m}$ of a previously received CTS/ACK frame, and a boolean variable $D$ to indicate if the channel quality is deteriorating or not.

Different data rates are supported by different RSS thresholds $\left(R_{\theta}\right)$. Therefore, like in DRA, each node stores a lookup table that maps different RSS thresholds to available data rates. Every time the sender receives a CTS, ACK, or NAK frame, it records $R_{m}$ of the moment and updates $D$. The sender adapts the retransmission rate depending on $R_{m}$ and $D$. Changes in the recorded RSS value indicates conditions in the wireless link between sender and receiver are changing, and it might be necessary to adapt the transmission rate $(r)$ accordingly. If $R_{m}$ is lower than $R_{\theta}$ for the current data rate $r$, or if it is significantly lower than the RSS values recorded earlier, $D$ is set to 1 (i.e. true); otherwise, it is set to 0 . Hence, $D$ indicates when the channel conditions are deemed as deteriorating.

Upon an ACK frame timeout, the sender checks $R_{m}$ and $D$. If $D$ is true, the sender determines that the wireless link has deteriorated and sets $r$ to $r_{\max \{1, r-1\}}$, which is the next available lower data rate. Otherwise, the sender determines that the loss is due to collision and a retransmission should be attempted at the current data rate $r$. Using the $D$ indicator, the sender can keep track of channel fluctuation even if the changes may not be large enough to cause DATA loss yet.

\section{NAK-Aided Loss Differentiation}

An RSS-based LD mechanism discussed above, suffers from the possibility of using outdated CTS/ACK's RSS value to

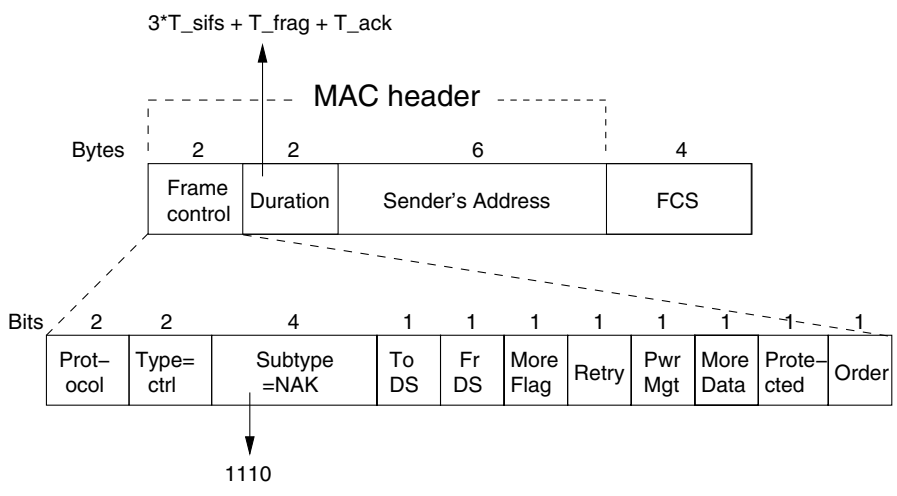

Fig. 1. Format of a NAK frame in DRANLD

infer link errors or collision if there is not sufficient recording. On the other hand, completely relying on NAK to diagnose frame losses must assume that ACK/NAK will always be received by the sender. However, such an assumption is unrealistic since a NAK frame can be lost as well. Therefore, a DRANLD sender must be able to handle the situation of missing any response for the receiver.

DRANLD combines the recorded RSS of CTS, ACK and NAK to effectively diagnose frame loss causes. If a NAK frame is received, the sender can easily infer DATA loss due to link errors. Similarly, if neither ACK nor NAK frame is received and if the recorded RSS value shows deteriorating channel quality, then the loss is caused by link errors. Only when neither ACK nor NAK is received and the channel quality has not been deteriorating does the sender infer frame loss due to collision.

The structure of the NAK frame is similar to that of an ACK frame (and other controls frames). The only difference being that of the frame type field which must have a one-bit difference to that of ACK. Since an RTS frame type field is set to 1011, CTS to 1100, and ACK to 1101, we suggest that the frame type field of a NAK frame be set to 1110 (as shown in Fig. 1). IEEE 802.11 specifies an Extended Inter-Frame Space (EIFS) that any receiving station must wait before starts its backoff timer when receiving an erroneous frame. EIFS includes SIFS, ACK and DIFS. However, since it takes the same time to transmit a NAK as an ACK, its duration field can similarly be set as that of an ACK frame. That is, the DURATION field of NAK is set to $S I F S+T_{\text {frag }}+S I F S+$ $T_{A C K}+$ SIFS.

It is not obvious that, in case of erroneous DATA frame, the receiver captures the address details of the transmitter. A station that sends out a DATA frame should remain in promiscuous mode waiting for an ACK from its intended receiver. If the receiver is not able to decode the header for sender's address, receiver's address field of the constructed NAK frame should be replaced with its own address (sender's address) so that a node in promiscuous mode knows itself is the intended recipient of the NAK frame. A node in promiscuous mode would have to listen to all the frames sent on the network rather than just the frames addressed to itself. 


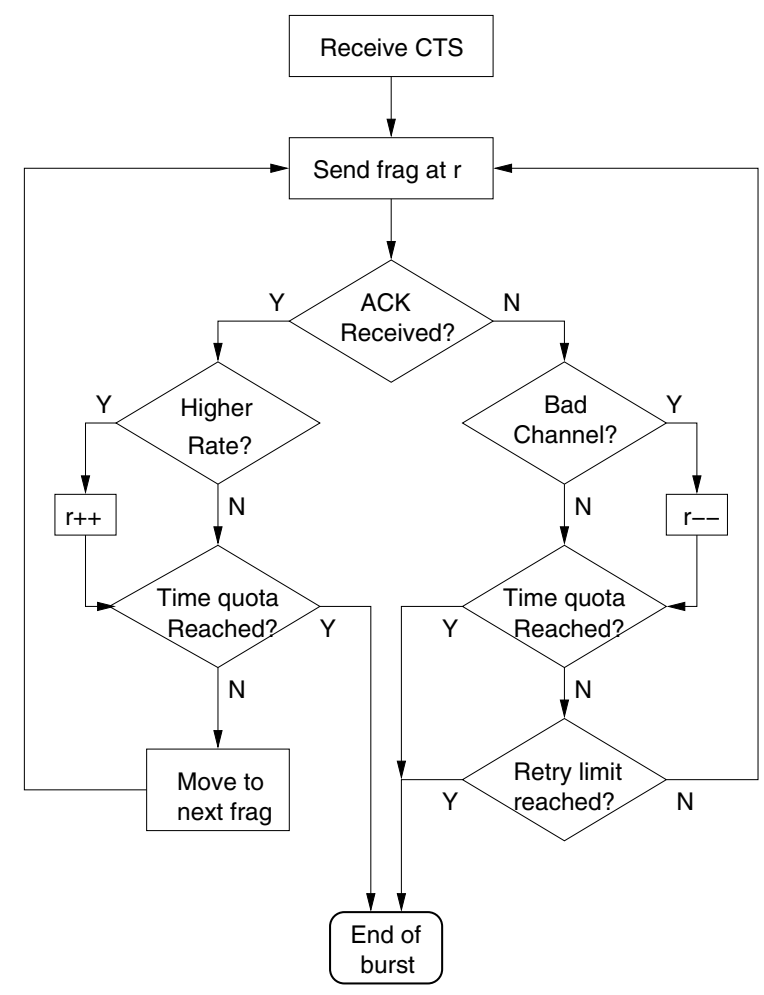

Fig. 2. DRANLD rate estimation and loss recovery strategy

\section{DRANLD Control Logic}

The sender only infers bad channel quality if it either receives a NAK frame or if its channel assessment indicates deteriorating channel quality (i.e. $D$ is 1 ). In such a case, retransmission is done at the next lower rate. Otherwise, if channel quality is appears good, the sender infers collision and retransmits at the current data rate. Fig. 2 summarizes the control logic for a DRANLD sending agent.

\section{Performance Evaluation}

We simulate DRANLD with $802.11 \mathrm{~b}$ (with data rates $1 \mathrm{Mbps}$, 2Mbps, 5.5Mbps, and 11Mbps) in ns-2. Simulation of other PHY with higher data rate is essentially the same. We use the precomputed time series of data of Punnoose et al. [13] to simulate a rapidly fading channel that follows the Ricean distribution. The Ricean Parameter $K$ is varied between 0 and 5 , to achieve different levels of contribution of the line-of-sight (LOS) component in the received signal. To investigate the effects of multiple transmitters and rapidly fading channel, we conduct our experiments in UDP traffic scenarios with varying nodes' mobility speed (maximum Doppler frequency $f_{m}$ ) and distance between the flows.

The simulation time is set to 50 seconds and the simulation is repeated 10 times for each value of $K$. The aggregate number of received frames for each value of $K$ is plotted. We compare DRANLD, DRA (our earlier work), and DRALD (DRANLD without NAK). Results obtained verifies our earlier findings that DRA performs better than OAR when the channel fluctuates more rapidly and performs similar to OAR in low or

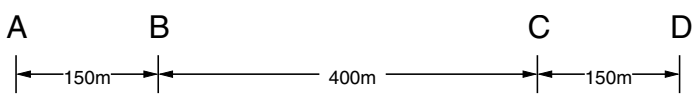

(a)

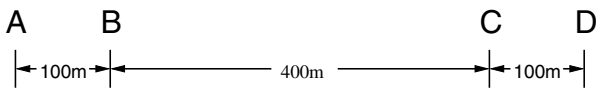

(b)

Fig. 3. DRALD simulation topologies

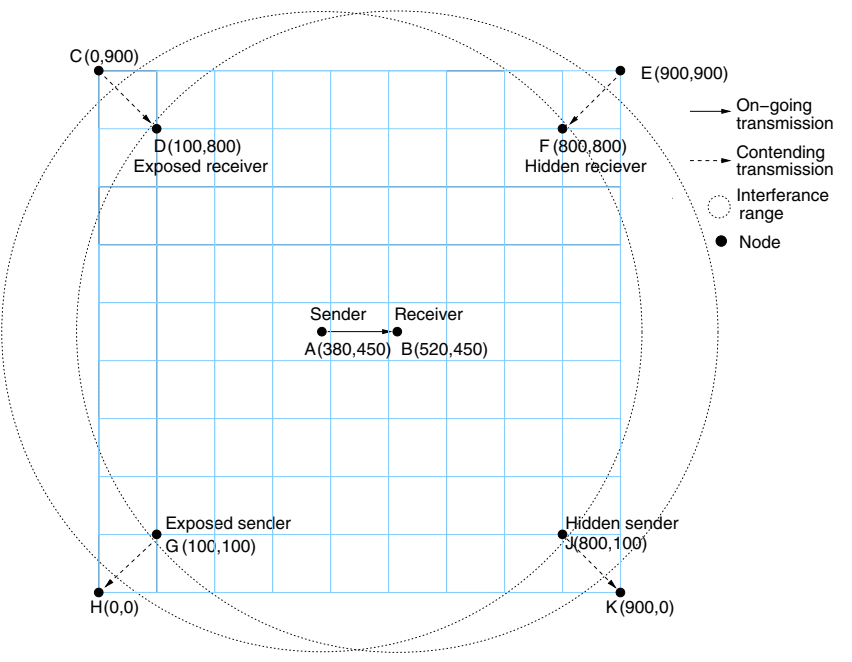

Fig. 4. DRANLD simulation topology

medium mobility rate. Additionally, we observe that DRALD benefits from its capability to adjust its transmission rate and frame burst. DRANLD achieves over 2\% throughput gain in a rapidly fluctuating channel over DRALD.

\section{A. Experiment Settings}

We test DRANLD's performance in two phases. First, we evaluate the performance of the RSS-based LD (DRALD) over our previous work DRA. Then, we evaluate the advantage of our NAK-aided loss differentiation mechanism (DRANLD) over DRALD, in slow and fast fluctuating channel.

1) DRALD versus DRA: Scenario 1: slow changing channel conditions. In the first scenario, shown in Fig. 3(a), we set maximum nodes' mobility speed to $2.5 \mathrm{~m} / \mathrm{s}$ and distance between each flow to $150 \mathrm{~m}$. These settings ensures a more stable channel with data rate fluctuating between $1 \mathrm{Mbps}$, 2Mbps and 5.5Mbps.

Scenario 2: rapidly changing channel conditions. The second scenario, shown in Fig. 3(b) assumes rapid channel fluctuations by setting nodes' mobility speed to $15 \mathrm{~m} / \mathrm{s}$. The distance between each flow is set to $100 \mathrm{~m}$ so that the data rates fluctuates between the all four rates.

2) DRANLD versus DRALD: Here, we evaluate the performance of DRANLD over a more representative UDP scenario with multiple hidden terminal flows, as illustrated in Fig. 4, which exhibits both path loss and interference. In the figure, nodes $D, F, G$ and $J$ are outside transmission range of 
sender $A$ and receiver $B$ but within their interference range. Therefore, RTS/CTS exchange between $A$ and $B$ prohibits nodes $G$ and $J$ from transmitting. However, nodes $C, E$, $H$ and $K$ are outside transmission and interference range of sender $A$ and receiver $B$. Thus, $C$ 's and $E$ 's transmission interferes with legitimate transmission between nodes $A$ and $B$.

The distance between individual flows is approximately $140 \mathrm{~m}$ and that between different flows is approximately $450 \mathrm{~m}$. Nodes mobility speed is varied between $2.5 \mathrm{~m} / \mathrm{s}$ and $15 \mathrm{~m} / \mathrm{s}$.

\section{B. Results Analysis}

We plot the cumulative number of frames received in Figures 5, 6, 9 and 10 in each scenario for all the values of $K$.

1) DRALD versus DRA: From Figures 5 and 6, we see that the performance of DRALD is better than that of its predecessors and its throughput increases as line-of-sight component $(K)$ becomes stronger. Generally, OAR and DRA performs poorly for $K=0$ and $K=1$; but for $K>1$ their performance is similar. We separate the flows and plot the cumulative frames received per second when $K=0$ and $K=1$ in Figures 7 and 8, respectively.

For $K=0$, OAR and DRA record a significant drop in cumulative frames received between $10-35$ seconds. Most of the transmitted DATA frames at this time are lost due to the rapidly fading channel and these schemes are not able to recover from the losses. When the channel quality improves, between $30-50$ seconds, the three protocols respond differently. DRA and DRALD senders benefit from their capability in adjusting data rate and number of fragments in a burst compared to OAR, which records improved performance for just a short period of time due to its shorter burst length.

On the other hand, for $K=1$, DRA performs slightly worse than OAR between $35-50$ seconds as illustrated in Fig. 8. Rapid channel fluctuations often occur for $K=1$. As such, when the channel quality improves rapidly, DRA is triggered to adapt to the changes, which means it must also ensure successful delivery of all the frames in its burst even when channel quality rapidly deteriorates.

DRALD recovers from frame losses by adjusting both data rate and remaining frames to transmit to match the current channel quality. Thus, it records a steady performance growth even for $K=0$ or $K=1$. As $K$ increases, the line-of-sight component is much stronger and the channel fluctuates less rapidly which enables steady data rates to be used over the simulation time for the three protocols.

2) DRANLD versus DRALD: Figures 9 and 10 plot DRANLD throughput gain, over DRALD and DRA, in slow and fast fluctuating channels respectively.

Both DRANLD and DRALD predict the channel quality at the receiver by recording the signal strength $\left(R_{m}\right)$ of a received CTS/ACK frame which they then use to determine if the quality is deteriorating or not. In a slow fluctuating channel, the recorded RSS value at the sender does not become obsolete quickly and is likely to efficiently predict the channel quality at the receiver. Thus, as shown in Fig. 9 DRANLD performance is similar to that of DRALD since additional reception of a NAK frame only confirms the channel quality already predicted using the existing LD mechanism.

However, Fig. 10 shows that DRANLD performs better than DRALD when channel conditions fluctuates more rapidly. The recorded RSS value quickly becomes obsolete in this scenario and may not predict the receiver's channel quality accurately. As such, additional reception of NAK frame indicates a fading channel at the receiver and the retransmission rate is adjusted accordingly.

\section{CONClusion AND Future Work}

This work proposes a NAK-aided loss differentiation mechanism, DRANLD, that incorporates three features for link adaptation in 802.11 wireless networks.

We consider an RSS-based LD mechanism, DRALD, that utilizes the signal strength of a received CTS/ACK frame to diagnose the cause of frame loss before selecting the retransmission rate. The RSS recordings enables the sender to predict channel quality, but it suffers from obsolete RSS recordings. This is especially possible in rapidly fluctuating channels. Thus, we built into DRALD a more versatile NAKaided loss differentiation mechanism, DRANLD. Reception of a NAK frame indicates a fading channel and a lower retransmission rate is be used. However, if neither an ACK nor a NAK frame is received by a DRANLD sender, the channel prediction is based on the previously recorded RSS value. Only when the channel is not deemed as deteriorating does the sender diagnose collision and the current data rate is used for retransmission. DRANLD records a throughput gain of over $2 \%$ in a rapidly fluctuating channel compared to RSSbased LD mechanism.

Using RTS/CTS frames to determine the initial burst lengths inevitably incurs overhead. Future work may involve using other techniques to determine the initial burst length and data rate for our rate adaptation mechanisms as well as exploring possibilities of using cross layer information in rate selection mechanisms.

\section{REFERENCES}

[1] “IEEE Standard 802.11," Wireless LAN Medium Access Control MAC and Physical Layer PHY Specifications, 1999.

[2] A. Kamerman and L. Monteban, "WaveLAN-II: A High-Performance Wireless LAN for the Unlicensed Band," Bell Labs Technical Journal, vol. 2, pp. $118-133,1997$.

[3] J. Kim, S. Kim, S. Choi, and D. Qiao, "CARA: Collision-Aware Rate Adaptation for IEEE 802.11 WLANs," in INFOCOM proceedings. 25th IEEE International Conference on Computer Communications, 2006, pp. 146-157.

[4] G. Holland, N. Vaidya, and P. Bahl, "A Rate-Adaptive MAC Protocol for Multi-Hop Wireless Networks," in Proceedings of the 7th annual international conference on Mobile computing and networking (MobiCom), 2001.

[5] B. Sadeghi, V. Kanodia, A. Sabharwal, and E. Knightly, "Opportunistic Media Access for Multirate Ad Hoc Networks," in Proceedings of the 8th annual international conference on Mobile computing and networking (MobiCom), 2002, pp. 24-35.

[6] S. Biaz and S. Wu, "OTLR: Opportunistic Transmission with Loss Recovery for WLANs," in IEEE Wireless Communications and Networking Conference (WCNC), 2008. 


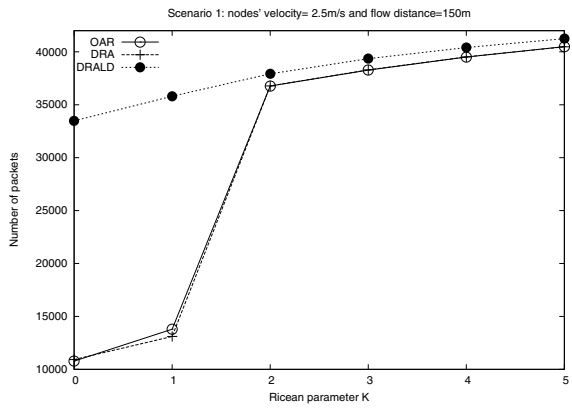

Fig. 5. Hidden flows each $150 \mathrm{~m}$ apart

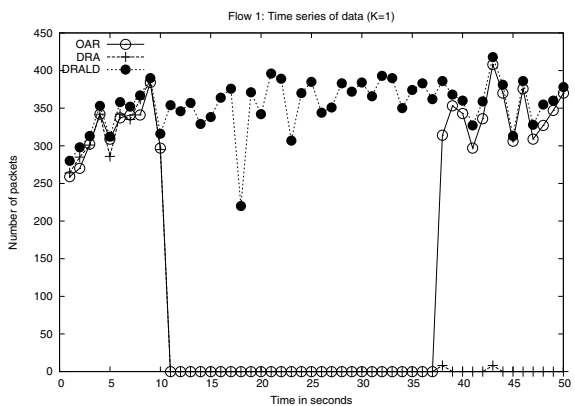

Fig. 8. Time series of data for $K=1$

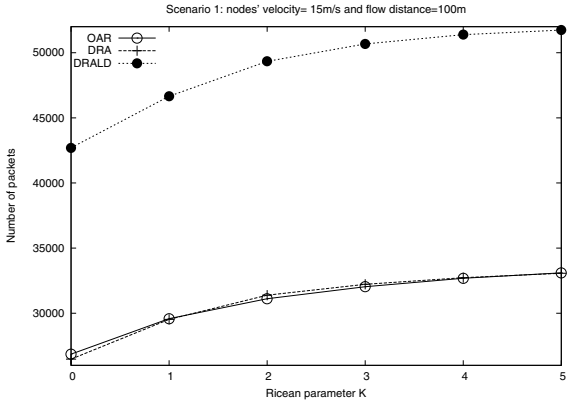

Fig. 6. Hidden flows each $100 \mathrm{~m}$ apart

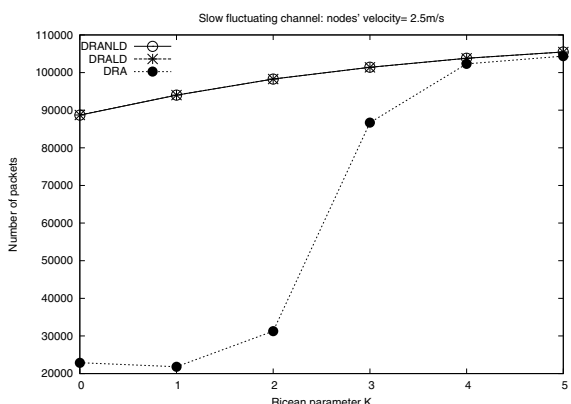

Fig. 9. Nodes mobility speed $=2.5 \mathrm{~m} / \mathrm{s}$

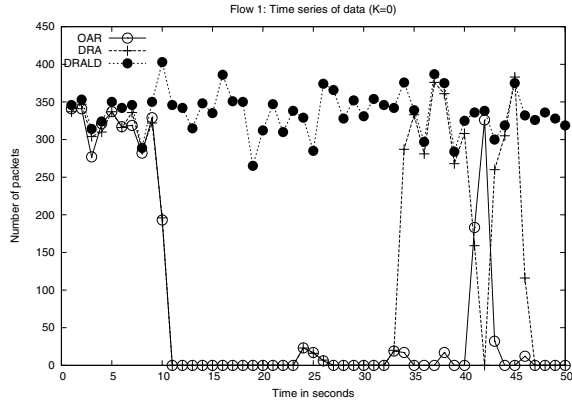

Fig. 7. Time series of data for $K=0$

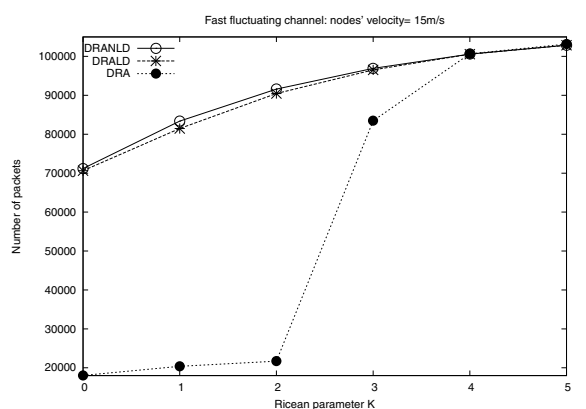

Fig. 10. Nodes mobility speed $=15 \mathrm{~m} / \mathrm{s}$

[7] _ , "Loss Differentiated Rate Adaptation in Wireless Networks," in IEEE Wireless Communications and Networking Conference (WCNC), 2008.

[8] Y. P. Chen, J. Zhang, and A. N. Ngugi, "An Efficient Rate-Adaptive MAC for IEEE 802.11," in Third International Conference on Mobile Ad-hoc and Sensor Networks (MSN), 2007.

[9] Q. Pang, V. Leung, and L. S. Chang, "A Rate Adaptation Algorithm for IEEE 802.11 WLANs Based on MAC-Layer Loss Differentiation," in Second International Conference on Broadband Networks (BroadNets), 2005, pp. 659-667.

[10] C.-C. Chen, H. Luo, E. Seo, N. H. Vaidya, and X. Wang, "Rate-adaptive framing for interfered wireless networks," in Proceedings of INFOCOM, 2007.

[11] C.-C. Chen, E. Seo, H. Kim, and H. Luo, "SELECT: Self-learning Collision Avoidance for Wireless Networks," in INFOCOM Proceedings. 25th IEEE International Conference on Computer Communications, 2006.

[12] K. Xu, M. Gerla, and S. Bae, "How Effective is the IEEE 802.11 RTS/CTS Handshake in Ad Hoc Networks," in Global Telecommunications Conference (GLOBECOM), 2002, pp. 72 - 76.

[13] R. J. Punnoose, P. V. Nikitin, and D. D. Stancil, "Efficient Simulation of Ricean Fading within a Packet Simulator," in Proceedings of 52nd Vehicular Technology Conference (VTC), 2000, pp. 764-767. 